\title{
Non-alcoholic fatty liver disease and lifestyle modifications, focusing on physical activity
}

\author{
Min-Sun Kwak ${ }^{1}$ and Donghee Kim²
}

\begin{abstract}
'Department of Internal Medicine, Healthcare Research Institute, Seoul National University Hospital Healthcare System Gangnam Center, Seoul, Korea; ${ }^{2}$ Division of Gastroenterology and Hepatology, Stanford University School of Medicine, Stanford, CA, USA
\end{abstract}

Received: October 9, 2017

Accepted: October 25, 2017

\section{Correspondence to}

Donghee Kim, M.D.

Division of Gastroenterology and Hepatology, Stanford University School of Medicine, 300 Pasteur Dr, Stanford, CA 94304, USA

Tel: +1-650-497-9261

Fax: +1-650-723-5488

E-mail: messmd@chol.com
Non-alcoholic fatty liver disease (NAFLD) is the most common chronic liver disease, and the prevalence of non-alcoholic steatohepatitis (NASH) with fibrosis is increasing as the population with NAFLD ages. To date, lifestyle modifications including weight loss, increased physical activity, and dietary changes remain the treatment of choice for NAFLD because there are no approved effective pharmacologic agents. Increased physical activity has therapeutic effects on NAFLD by reducing hepatic fat independent of weight reduction. Indeed, even minimal physical activity below the recommended threshold may have a beneficial impact on NAFLD. Aerobic activity and resistance training have similar effects on NAFLD. Universal recommendations for the optimal intensity and dose of physical activity have not been established. Therefore, physical activity should be tailored based on a patient's clinical characteristics, comorbidities, and fitness capacity. Physical activity also prevents the development of NAFLD and may represent a valuable strategy for reducing the public health burden. However, there are insufficient data supporting the effects of physical activity on the progression of non-alcoholic fatty liver to NASH with advanced fibrosis, and on extrahepatic disease-related morbidity and mortality. In this paper, we review the role of physical activity in the management of NAFLD.

Keywords: Hepatic steatosis; Exercise; Life-style modification

\section{INTRODUCTION}

Non-alcoholic fatty liver disease (NAFLD) is the most common chronic liver disease worldwide and is estimated to affect one-third of the general population $[1,2]$. It includes a spectrum of diseases ranging from non-alcoholic fatty liver (NAFL), non-alcoholic steatohepatitis (NASH), advanced fibrosis, and end-stage liver disease to hepatocellular carcinoma [3]. The increasing prevalence of obesity and diabetes parallels the increasing prevalence and severity of NAFLD, and as the population ages, a considerable number of patients with NAFLD will develop cirrhosis and end-stage liver disease. In addition, NAFLD is closely associated with cardiovascular disease, diabetes, and chronic kidney disease, which eventually lead to increased cardiovascular mortality [4]. There is consistent evidence supporting common pathophysiologic mechanisms between metabolic syndrome and NAFLD, which usually involve visceral obesity and insulin resistance [5-7]. However, there are currently no approved pharmacologic therapies for NAFLD. Therefore, similar to the recommended treatment for metabolic syndrome, the treatment for NAFLD has focused on lifestyle modifications including weight loss, dietary changes, and increased physical activity [8]. In this review, we summarize the lifestyle modifications recommended for patients with NAFLD, focusing on the impact of physical activity. 


\section{DIAGNOSIS AND EPIDEMIOLOGY OF NAFLD}

NAFLD is defined as hepatic fat accumulation ( $\geq 5 \%$ of the liver) determined either by imaging or histology after excluding other causes of hepatic steatosis such as viral hepatitis, significant alcohol consumption, use of steatogenic medication, or hereditary disorders [9]. NAFLD includes NAFL, which is defined as hepatic steatosis without hepatocellular injury, and NASH, which is defined as hepatic steatosis with hepatocyte balloon degeneration and/or hepatic lobular inflammation [10,11]. The worldwide prevalence of NAFLD is reportedly $20 \%$ to $30 \%$ in the general population, and its incidence is approximately 20 to 30 per 1,000 person-years depending on their characteristics [11]. In Korea, the prevalence of ultrasonography-diagnosed NAFLD is approximately $16 \%$ to $33 \%$, which is increasing in parallel with the increasing prevalence of obesity $[10,12]$. A recent meta-analysis estimated the prevalence of NASH to be $1.5 \%$ to $6.5 \%$ in the general population, but data are scarce [13]. A recent Markov model for forecasting NAFLD progression indicated that NASH- and NAFLD-related end-stage liver disease and mortality will exponentially increase in the United States due to the increasing prevalence of obesity and diabetes and the ageing population [14]. Old age, diabetes, dyslipidemia, obesity, and metabolic syndrome are known risk factors for NAFLD and are closely associated with NASH with advanced fibrosis $[9,10,15]$. The increasing prevalence of NASH with advanced fibrosis is concerning due to the associated liver-related and cardiovascular disease-related morbidity and mortality [16]. NASH is also associated with an increased risk for liver cirrhosis and hepatocellular carcinoma. Overall, $10 \%$ to $29 \%$ of patients with NASH progress to liver cirrhosis within 10 years, and $4 \%$ to $27 \%$ of these patients have hepatocellular carcinoma [17]. Two imaging methods, magnetic resonance elastography $[18,19]$ and transient elastography $[20,21]$, are useful for diagnosing advanced fibrosis and may be useful for detecting NASH with or without advanced fibrosis. In the clinical setting, several non-invasive serum algorithms for diagnosing advanced fibrosis in patients with NAFLD have been developed in the past several years. Of these various algorithms, the FIB-4 (fibrosis-4) and NAFLD fibrosis scores are the most widely validated, and have superior test characteristics [22-24].

\section{NAFLD AND CARDIOVASCULAR DISEASE}

There is accumulating evidence that NAFLD is related to non-liver systemic complications such as cardiovascular disease [25-27], diabetes [28], and metabolic syndrome [29,30], which eventually leads to increased cardiovascular mortality [4,31]. The most plausible pathophysiologic factors underlying the relationship between NAFLD and systemic extrahepatic complications are insulin resistance and visceral obesity [5,32]. It is difficult to verify the causal relationships among visceral obesity, insulin resistance, and NAFLD because of the associations among these factors. However, an expanded and inflamed visceral fat mass releases various proinflammatory molecules that are associated with insulin resistance and atherosclerosis [7,33,34]. Insulin resistance and excessive triglycerides, particularly those from visceral fat, are pathogenic factors in the development and progression of NAFLD [7,33,34]. All of these factors are responsible for chronic inflammation, oxidative stress, and lipotoxicity, which can lead to a hypercoagulated state, atherogenic dyslipidemia, and dysglycemia; in turn, these factors provoke systemic extrahepatic complications of NAFLD such as cardiovascular disease and diabetes [34,35].

\section{RECOMMENDED LIFESTYLE MODIFICATIONS FOR TREATING NAFLD (WEIGHT LOSS AND DIETARY CHANGES)}

To date, there are no approved, effective pharmacological treatments for NAFLD. Therefore, treatments have focused on lifestyle modifications such as weight loss, dietary control, and increased physical activity [9,36], similar to the recommendations for metabolic syndrome $[4,8]$. The treatment strategy for individual components of metabolic syndrome may also be applied for NAFLD, with a focus on the control of blood glucose, blood pressure, triglycerides, and high density lipoprotein cholesterol. Current practice guidelines for metabolic syndrome from the American Heart Association/ National Heart, Lung, and Blood Institute Scientific Statement recommend reducing body weight by $7 \%$ to 10\% from baseline [8]. The guidelines for dietary changes recommend reducing the intake of saturated fat to < $7 \%$ of total calories, reducing trans-fat intake, and main- 
Table 1. Weight reduction and dietary restriction guidelines

\begin{tabular}{|c|c|c|}
\hline Study & Weight reduction & Dietary restriction \\
\hline AASLD [11] & $\begin{array}{l}3 \%-5 \% \text { Reduction } \rightarrow \text { improve steatosis } \\
7 \%-10 \% \text { Reduction } \rightarrow \text { improve most of the } \\
\text { histopathologic features of NASH, including } \\
\text { fibrosis }\end{array}$ & $\begin{array}{l}\text { Hypocaloric diet (daily reduction by 500-1,000 } \\
\text { kcal) } \\
\text { Specific macronutrient composition: requires } \\
\text { verification } \\
\text { Mediterranean diet: seems beneficial }\end{array}$ \\
\hline EASL-EASD-EASO [36] & $7 \%-10 \%$ Reduction & $\begin{array}{l}\text { 500-1,000 kcal energy reduction to induce weight } \\
\text { loss of } 500-1,000 \mathrm{~g} / \mathrm{wk} \\
\text { Low-to-moderate fat and moderate-to-high } \\
\text { carbohydrate intake } \\
\text { Low-carbohydrate ketogenic diets or high-protein } \\
\text { diets } \\
\text { Avoid fructose-containing beverages and foods }\end{array}$ \\
\hline KASL [10] & $7 \%-10 \%$ Reduction & $\begin{array}{l}\text { 400-500 kcal energy reduction } \\
\text { Low-carbohydrate diet and low-fructose diet }\end{array}$ \\
\hline
\end{tabular}

AASLD, American Association for the Study of Liver Diseases; NASH, non-alcoholic steatohepatitis; EASL-EASD-EASO, European Association for the Study of the Liver-European Association for the Study of Diabetes-European Association for the Study of Obesity; KASL, Korean Association for the Study of the Liver.

taining dietary cholesterol intake at $<200 \mathrm{mg} /$ day and total fat at $25 \%$ to $35 \%$ of total calories [8]. Observational studies have shown that increasing physical activity is beneficial, and that there is a dose-response relationship between physical activity and metabolic syndrome [37]. Engaging in regular moderate-intensity physical activity for at least 30 minutes for more than 5 days/week is recommended for the treatment of metabolic syndrome $[8,38,39]$.

For patients with NAFLD, lifestyle modifications including weight loss, total calorie restriction, and increased physical activity are also recommended, consistent with the recommendations for metabolic syndrome. The characteristics of each guideline for weight reduction and dietary restrictions for the treatment of NAFLD are summarized in Table 1. Weight loss with lifestyle modification is the most important goal for improving NAFLD. The American Association for the Study of Liver Diseases (AASLD) NAFLD practice guidelines recommend body-weight reduction of at least $3 \%$ to $5 \%$ to improve hepatic steatosis, and greater body-weight reduction ( $\geq 7 \%$ ) to improve the histologic features of NASH including fibrosis [11]. A prospective study of the histology of paired liver samples showed that weight loss improved both NASH-related factors and fibrosis. In addition, a dose-response relationship was evident between weight loss and histologic NASH parameters [40]. Guidelines from the European Association for the Study of the Liver-European Association for the Study of Diabetes-European Association for the Study of Obesity (EASL-EASD-EASO) and Korean Association for the Study of the Liver (KASL) [10] similarly recommend a weight reduction of $7 \%$ to $10 \%$ for the improvement of NAFLD.

Regarding diet, the EASL-EASD-EASO and AASLD guidelines recommend calorie restriction by 500 to $1,000 \mathrm{kcal}$ to induce weight loss of 0.5 to $1.0 \mathrm{~kg} /$ week with a target total body weight reduction of $7 \%$ to $10 \%$. The KASL guidelines recommend energy reduction of 400 to $500 \mathrm{kcal}$, which is slightly lower than that in the other two guidelines. Concerning macronutrient composition, the EASL-EASD-EASO guidelines recommend a low-to-moderate fat intake and a moderate-to-high carbohydrate intake or low-carbohydrate ketogenic diets or high-protein diets [36]; however, the AASLD guidelines do not recommend a specific macronutrient composition, as it appears to be less relevant than the end result of sustained weight loss [11]. Both the AASLD and EASL-EASD-EASO guidelines report the beneficial effects of the Mediterranean diet. The KASL guidelines suggest a low carbohydrate diet, considering the higher daily carbohydrate intake in Korea compared to other areas [10]. 
Table 2. Physical activity guidelines

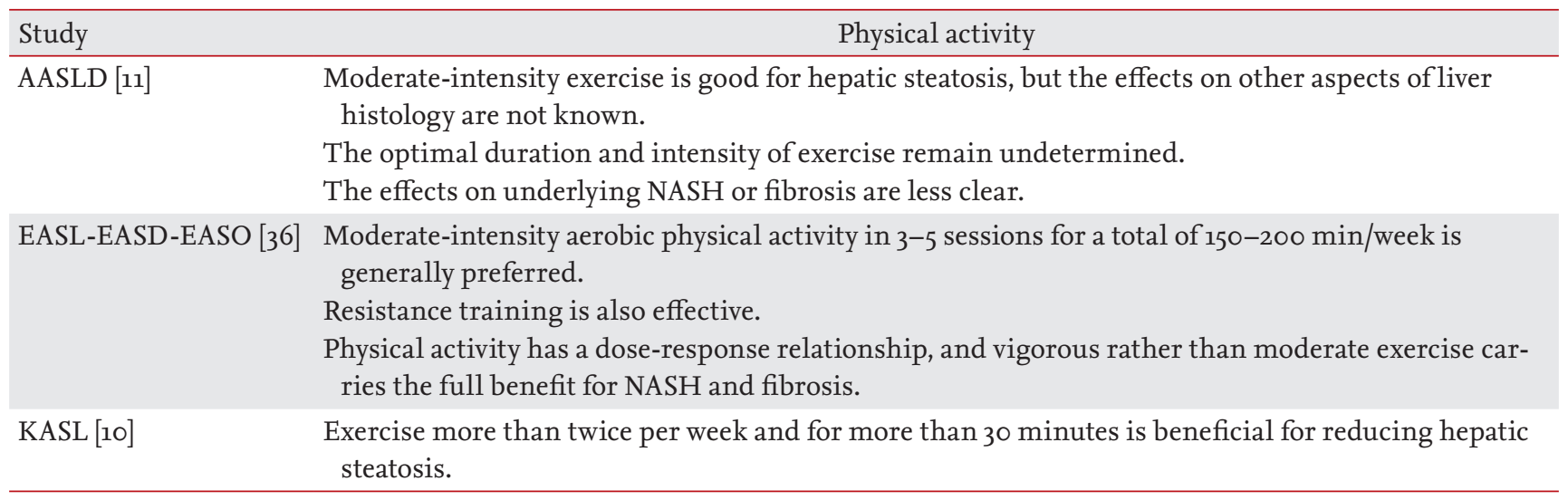

AASLD, American Association for the Study of Liver Diseases; NASH, non-alcoholic steatohepatitis; EASL-EASD-EASO, European Association for the Study of the Liver-European Association for the Study of Diabetes-European Association for the Study of Obesity; KASL, Korean Association for the Study of the Liver.

\section{RECOMMENDATIONS ON PHYSICAL ACTIVITY FOR TREATING NAFLD}

Regarding physical activity as a lifestyle modification, increasing physical activity reduces intrahepatic triglyceride content and markers of hepatocellular injury in patients with NAFLD independent of weight loss (Table 2) [41-45]. Specifically, 150 to $200 \mathrm{~min} /$ week of moderate-intensity aerobic physical activity in three to five sessions are recommended by the EASL-EASD-EASO guidelines [36]. The KASL practice guidelines recommend at least 30 minutes of physical activity more than twice per week to reduce hepatic fat [10]. The AASLD guidelines suggest the beneficial effects of physical activity, but do not state a specific cut-off for physical activity. In this review, we focus on the relationship between physical activity as a lifestyle modification and NAFLD.

\section{THERAPEUTIC EFFECTS OF PHYSICAL ACTIVITY ON IMPROVEMENTS IN NAFLD}

\section{Definition of terms}

Physical activity is defined as body movement produced by skeletal muscles, which results in energy expenditure beyond that at rest [46]. Exercise is a subset of physical activity that is planned, structured, and repetitive, with the goal of improving or maintaining physical fitness [46]. Whereas most previous studies have utilized phys- ical activity mixed with exercise, we only used 'physical activity' with a broader meaning in our review. The relationship between dose and intensity of physical activity is important when defining the amount of physical activity. In this review, we defined the term 'dose' as the total amount of energy expended in physical activity [46]. The frequency of physical activity has been considered the dose of physical activity in several studies, although the frequency does not precisely reflect the dose. The intensity of physical activity refers to the rate of energy expenditure during such activity $[46,47]$, and can be defined in either absolute or relative terms. Absolute intensity reflects the rate of energy expenditure during physical activity and is usually expressed in metabolic equivalents (METs). One MET is a resting metabolic rate of approximately $3.5 \mathrm{~mL} \mathrm{O}_{2} / \mathrm{kg} / \mathrm{min}$. The relative intensity refers to the percentage aerobic power utilized during physical activity and is expressed as the percentage of maximal heart rate or of the maximal aerobic capacity ( $\mathrm{VO}_{2}$ max). Moderate-intensity physical activity is defined in terms of relative intensity as $40 \%$ to $60 \%$ of $\mathrm{VO}_{2}$ max, and in terms of absolute intensity as 4 to 6 METs. Vigorous-intensity physical activity is defined as relative intensity more than $60 \%$ of the $\mathrm{VO}_{2}$ max or absolute intensity $>6$ METs [48]. In the widely used International Physical Activity Questionnaire, moderate-intensity activity was defined as 4 METs, and vigorous-intensity activity was considered 8 METs [49]. 


\section{Aerobic versus resistance physical activity}

One practical issue regarding physical activity is determining which type of physical activity (aerobic vs. resistance) has greater beneficial effects on NAFLD. Both now and in the past, aerobic physical activity is highly recommended to improve markers of hepatic injury such as aspartate aminotransferase and alanine aminotransferase levels, and histologic features of NAFLD such as the NAFLD activity score [42,50-52]. In two recent randomized trials that compared the impact of aerobic activity and resistance training on NAFLD, there were no significant differences between these two types of physical activity regarding improvements in hepatic injury or intrahepatic triglycerides [53,54], despite a previous report [55] stating that aerobic physical activity leads to a greater reduction in hepatic fat level. Hallsworth et al. [56] first reported that resistance training specifically improves NAFLD independent of body weight change compared to standard treatment. Eight weeks of resistance training elicited a 13\% relative reduction in the intrahepatic triglyceride level $(p<0.05)$ [56]. Bacchi et al. [53] performed a randomized clinical trial comparing aerobic training and resistance training to reduce intrahepatic triglyceride levels in patients with type 2 diabetes, and reported a similar reduction in both groups. NAFLD similarly regressed in about one-quarter of the patients in both of the physical activity groups ( $23.1 \%$ vs. $23.5 \%, p$ $=0.99$ ) [53]. A recent systematic review comparing aerobic activity and resistance training showed similar improvements in NAFLD between resistance and aerobic physical activity [42,50]. Aerobic activity and resistance training seem to have different mechanisms for improving NAFLD. Aerobic physical activity improves NAFLD by activating lipolysis in various tissues, upregulating uncoupling protein-1 and peroxisome proliferator-activated receptor $\gamma$ pathways, and altering adipokine levels [50]. Resistance training may improve NAFLD by evoking hypertrophy of type II muscle fibers, altering myokine levels, and activating glucose transporter 4, AMP-activated protein kinase, and caveolins [50]. A recent study showed that resistance training improved NAFLD with less energy consumption [50]. Close associations between sarcopenia and NASH and advanced fibrosis in NAFLD also suggest that resistance training may have effects on NASH and advanced fibrosis in patients with NAFLD and sarcopenia [57]. Thus, patients with NAFLD can perform their preferred physical activity, aerobic activity or resistance training, and resistance training could be recommended for those with poor cardio-respiratory fitness or sarcopenia, or those who cannot tolerate aerobic physical activity [50].

\section{Minimum dose of physical activity for NAFLD}

The beneficial effects of minimal levels of physical activity on NAFLD are unclear. Randomized clinical trials have usually applied the recommended dose of physical activity in the intervention arm; thus, it is difficult to determine the impact of minimum levels of physical activity, below that recommended, on NAFLD based on current evidence. A recent randomized trial evaluated the effects of 8 weeks of different doses and intensities of aerobic physical activity on liver fat levels using the following groups: group 1 (low-to-moderate intensity, high dose: $50 \% \mathrm{VO}_{2 \text { peak }}, 60$ minutes, 4 days/week), group 2 (high intensity, low dose: $70 \% \mathrm{VO}_{\text {2peak }}, 45$ minutes, 3 days/week), group 3 (low-to-moderate intensity, low dose: $50 \% \mathrm{VO}_{2 \text { peak }}, 45$ minutes, 3 days/week), and group 4 (placebo). In this study, all of the groups, even group 3 with the lowest physical activity dose and intensity, showed a reduction in liver fat and visceral fat without significant weight loss [58]. A Korean cross-sectional study showed that an increased sitting time (sedentary lifestyle) was associated with a high prevalence ratio of NAFLD (prevalence ratio: 1.04 for 5 to 9 hours/day and 1.09 for $\geq 10$ hours/day compared to $<5$ hours/day of sitting time) [59]. The risk for NAFLD decreased by $6 \%$ in the minimally active groups compared to the inactive group [59]. Given the paucity of evidence, these results suggest that even minimal engagement in physical activity might provide a slight benefit to NAFLD patients. It may be worth recommending that NAFLD patients who are reluctant to engage in physical activity "just be active," irrespective of weight change or the amount of physical activity.

\section{Dose-response relationship between physical activ- ity and NAFLD}

While minimal physical activity and the avoidance of sedentary behavior may be slightly beneficial for NAFLD as described above, several studies have suggested a dose-response relationship between physical activity and improvements in NAFLD. Recently, we report- 
ed a dose-dependent inverse association between the dose of physical activity and prevalence of NAFLD, independent of visceral adipose tissue area and insulin resistance ([fourth quartile (highest physical activity): odds ratio (OR), o.68; 95\% confidence interval (CI), 0.54 to 0.85 ]; [third quartile: OR, $0.74 ; 95 \% \mathrm{CI}, 0.59$ to 0.93 ] vs. first quartile [lowest physical activity]; $p$ for trend < 0.001 ) [6o]. Another Korean cross-sectional study also reported a dose-response relationship between physical activity and NAFLD. The prevalence ratios for NAFLD between minimally active and health-enhancing physically active groups and the inactive group were 0.94 (95\% CI, 0.92 to 0.95 ) and 0.80 (95\% CI, 0.78 to 0.82 ), respectively ( $p$ for trend < 0.001) [59]. The lower cut-off for recommended physical activity according to the Department of Health and Human Services (DHHS) and the US Department of Agriculture (USDA) for adults is $\geq 150 \mathrm{~min} /$ week of moderate activity or $\geq 75 \mathrm{~min} /$ week of vigorous activity, which is approximately 500 MET-min/week [51]. Subjects who satisfied the minimum recommended physical activity ( $\geq 500 \mathrm{MET}$-min/week) had a 34\% decreased risk for NAFLD compared to inactive subjects, which implies that the typical recommended dose of regular physical activity (approximately $\geq 500 \mathrm{MET}-\mathrm{min} /$ week) is beneficial [6o]. A recent retrospective longitudinal study showed that subjects with more than $250 \mathrm{~min} /$ week of moderate-to-vigorous physical activity had significantly attenuated levels of hepatic steatosis ( $-31.8 \%$ vs. $-23.2 \%)$ compared to those with fewer than $250 \mathrm{~min} /$ week of moderate-to-vigorous physical activity independent of detectable weight reduction [43]. All of these data support a dose-response relationship between NAFLD and physical activity.

\section{An optimal physical activity protocol for NAFLD}

Although a dose of physical activity below the current recommendations may have slight beneficial effects on NAFLD, accumulating evidence suggests an inverse dose-response relationship between physical activity and NAFLD. However, the optimal protocol in terms of type, intensity, and dose of physical activity for the treatment of NAFLD has not been established. Because current evidence on the intensity and dose of physical activity was obtained from diverse and heterogeneous populations regarding age, sex, ethnicity, and medical comorbidities, it is difficult to make a conclusive recom- mendation for everyone. Therefore, the optimal training protocol in terms of type (aerobic vs. resistance), intensity, and dose should be tailored based on a patient's clinical characteristics, comorbidities, fitness status, and preferences [36].

\section{Effects of physical activity on NASH or advanced fibrosis}

Most studies have focused on the effects of physical activity on NAFL because of the difficulty in diagnosing NASH and advanced fibrosis without performing an invasive liver biopsy. However, because NASH with advanced fibrosis is closely associated with an increased risk for liver-related and non-liver-related mortality [16], the effects of physical activity on NASH or advanced fibrosis are important for reducing the public health risk. A cross-sectional study by the NASH Clinical Research Network showed that subjects who met the vigorous physical activity recommendations of the DHHS and USDA ( $\geq 6 \mathrm{MET}$, minimum target of $75 \mathrm{~min} /$ week), but not those who met the moderate physical activity recommendations ( 3 to 5.9 MET, minimum target of 150 $\mathrm{min} /$ week), were less likely to have NASH (OR, o.65; 95\% CI, 0.43 to 0.98). Doubling the recommended time spent in vigorous physical activity, which is suggested for achieving additional health benefits, is associated with a decreased adjusted OR for advanced fibrosis (OR, 0.53; 95\% CI, 0.29 to 0.97 ) [51]. A small pilot study comparing the effects of physical activity versus diet showed no significant reduction in NAFLD activity score or fibrosis or in NASH resolution rate in the physical activity intervention arm (6 months of circuit physical activity training in nine patients with NAFLD) compared to the dietary intervention arm [61]. The effects of physical activity per se on histologic changes could not be evaluated due to the lack of a control group [61]. A recent randomized controlled trial including 24 biopsy-proven NASH patients showed that 12 weeks of cycling and resistance training without weight loss reduced intrahepatic triglyceride content and plasma triglycerides. However, there were no effects on metabolic parameters, circulating levels of markers of inflammation (interleukin 6 , tumor necrosis factor $\alpha$, and C-reactive protein), and levels of non-invasive markers of fibrosis [52]. One study of 59 NASH subjects (NASH was diagnosed by histology in fewer than 30 subjects) showed significantly greater 
normalization of serum alanine aminotransferase levels in patients in the moderate-intensity aerobic physical activity intervention arm compared to those without an exercise program [62]. Current and previous randomized trials and longitudinal studies have been too small or of inadequate duration to draw a conclusion regarding the effects of physical activity on NASH and advanced fibrosis. To more accurately determine the effects of physical activity on NASH and advanced fibrosis, larger and longer-term studies are warranted. Until further evidence regarding the effects of physical activity on NASH and advanced fibrosis is obtained, lifestyle modifications including weight loss, dietary changes, and physical activity should be recommended for patients with NASH and advanced fibrosis who are considering pharmacologic treatment [63].

\section{Therapeutic effects of physical activity on extrahe- patic manifestations of NAFLD}

The primary outcome of most studies regarding the effects of physical activity on NAFLD was an improvement in hepatic steatosis or levels of surrogate markers of hepatic injury, inflammation, or fibrosis. Although liver-related mortality is increased in patients with NAFLD, particularly in those with NASH or advanced fibrosis, the overall mortality of NAFLD patients is mainly due to cardiovascular disease [4]. Accumulating evidence from general population-based studies shows that physical activity reduces cardiovascular disease mortality in the general population $[46,64,65]$. Physical activity capacity also predicted cardiovascular disease mortality in a clinically healthy population $[66,67]$. A meta-analysis showed that an improvement in $\mathrm{VO}_{2}$ max of 1 MET reduced all-cause mortality by $13 \%$ and cardiovascular events by $15 \%$ among healthy participants $[63,68]$. Physical activity also had beneficial effects on established cardiovascular risk factors such as insulin resistance, elevated blood pressure, glucose intolerance, elevated triglycerides, obesity, and low high-density lipoprotein cholesterol levels, which are also closely associated with NAFLD [46,69-72]. In fact, a randomized controlled trial of subjects with biopsy-proven NASH found decreased plasma triglyceride levels in the physical activity arm compared to the control arm [52]. To sum up these results, physical activity may play roles in the improvement of both NAFLD and extrahepatic manifestations, such as metabolic abnormalities and cardiovascular-related morbidity.

\section{THE PREVENTIVE EFFECTS OF PHYSICAL AC- TIVITY ON NAFLD DEVELOPMENT AND PRO- GRESSION}

Currently, in the absence of approved, effective pharmacologic treatments for NAFLD, which is a highly prevalent and emerging health burden, treatments focused on lifestyle modifications and prevention strategies are crucial for reducing the future public health burden. In our longitudinal cohort study, which included 1,373 subjects without NAFLD at baseline, 288 (21.0\%) developed NAFLD during a median 4.4-year follow-up period. A higher dose of physical activity at baseline was associated with a decrease in subsequent NAFLD development [73]. Compared to subjects with the lowest dose of physical activity, those who reported the highest dose of physical activity had a $34 \%$ decreased risk for developing NAFLD, even after adjustment for visceral obesity and insulin resistance [73]. Furthermore, there was a dose-response relationship between the level of physical activity (evaluated as MET-min/week) and incident NAFLD ( $p$ for trend $=0.025$ ). A recent Korean study reported similar results during a 5-year follow-up, although physical activity was only measured as frequency per week [74]. A Japanese prospective cohort study showed that only vigorous-intensity physical activity ( $\geq 7 \mathrm{METs}$ ), not moderate-to-low (3 to $5 \mathrm{METs}$ ) or moderate-to-high (5 to $7 \mathrm{METs}$ ) intensity physical activity, prevented the progression of NAFL to NASH, which is defined as NAFLD with elevated liver enzyme levels [75]. In a recent study in Taiwan, physical activity > $150 \mathrm{~min} /$ week was significantly protective against cirrhosis in obese patients, although patients with NAFLD or other chronic liver diseases were included in the study [76].

In daily life, patients are left with the challenge of physical activity, which is difficult to implement or maintain in a predictable fashion. We evaluated whether changes in the physical activity dose during the median 4.4-year follow-up affected new development of NAFLD in subjects without NAFLD at baseline [73]. As expected, subjects with reduced physical activity during follow-up had a 59\% increased risk for incident NAFLD compared 
to subjects with sustained or increased physical activity (hazard ratio, 1.59; 95\% CI, 1.11 to 2.27; $p=0.011$ ). This result suggests that sustained physical activity is important for the prevention of NAFLD.

\section{CONCLUSIONS}

Increasing physical activity has beneficial effects on NAFLD by improving hepatic injury, hepatic fat, and histologic features of NAFLD, independent of weight loss. Even a level of physical activity below that recommended or the avoidance of a sedentary lifestyle may have a beneficial impact on NAFLD, emphasizing the importance of "just being active." Aerobic physical activity and resistance training showed similar improvements in NAFLD. While there is a dose-response relationship between physical activity and NAFLD and sustained physical activity has beneficial effects on NAFLD, universal recommendations on the optimal intensity and dose of physical activity have not been established. Therefore, physical activity should be tailored based on a patient's clinical characteristics, medical comorbidities, and physical fitness capacity. The beneficial effects of physical activity on NAFLD development could be applied to prevent incident NAFLD and reduce the future public health burden. However, evidence regarding the effects of physical activity on NASH with advanced fibrosis and on non-liver disease-related morbidity and mortality is insufficient and must be augmented and verified in future studies.

\section{Conflict of interest}

No potential conflict of interest relevant to this article was reported.

\section{REFERENCES}

1. Sayiner M, Koenig A, Henry L, Younossi ZM. Epidemiology of nonalcoholic fatty liver disease and nonalcoholic steatohepatitis in the united states and the rest of the world. Clin Liver Dis 2016;20:205-214.

2. Younossi ZM, Stepanova M, Afendy M, et al. Changes in the prevalence of the most common causes of chronic liver diseases in the United States from 1988 to 2008. Clin
Gastroenterol Hepatol 2011;9:524-530.

3. Satapathy SK, Sanyal AJ. Epidemiology and natural history of nonalcoholic fatty liver disease. Semin Liver Dis 2015;35:221-235.

4. Kwak MS, Kim D. Long-term outcomes of nonalcoholic fatty liver disease. Curr Hepatol Rep 2015;14:69-76.

5. Paschos P, Paletas K. Non alcoholic fatty liver disease and metabolic syndrome. Hippokratia 2009;13:9-19.

6. Kwon H, Kim D, Kim JS. Body fat distribution and the risk of incident metabolic syndrome: a longitudinal cohort study. Sci Rep 2017;7:10955.

7. Kim D, Chung GE, Kwak MS, et al. Body fat distribution and risk of incident and regressed nonalcoholic fatty liver disease. Clin Gastroenterol Hepatol 2016;14:132-138.

8. Grundy SM, Cleeman JI, Daniels SR, et al. Diagnosis and management of the metabolic syndrome: an American Heart Association/National Heart, Lung, and Blood Institute Scientific Statement. Circulation 2005;112:2735-2752.

9. Chalasani N, Younossi Z, Lavine JE, et al. The diagnosis and management of non-alcoholic fatty liver disease: practice guideline by the American Association for the Study of Liver Diseases, American College of Gastroenterology, and the American Gastroenterological Association. Hepatology 2012;55:2005-2023.

10. Korean Association for the Study of the Liver (KASL). KASL clinical practice guidelines: management of nonalcoholic fatty liver disease. Clin Mol Hepatol 2013;19:325348.

11. Chalasani N, Younossi Z, Lavine JE, et al. The diagnosis and management of nonalcoholic fatty liver disease: practice guidance from the American Association for the Study of Liver Diseases. Hepatology 2017 Jul 17 [Epub]. https://doi.org/10.1002/hep.29367.

12. Chung GE, Kim D. Epidemiology of nonalcoholic fatty liver disease. Korean J Med 2014;86:399-404.

13. Younossi ZM, Koenig AB, Abdelatif D, Fazel Y, Henry L, Wymer M. Global epidemiology of nonalcoholic fatty liver disease-meta-analytic assessment of prevalence, incidence, and outcomes. Hepatology 2016;64:73-84.

14. Estes C, Razavi H, Loomba R, Younossi Z, Sanyal AJ. Modeling the epidemic of nonalcoholic fatty liver disease demonstrates an exponential increase in burden of disease. Hepatology 2017 Aug 12 [Epub]. https://doi. org/10.1002/hep.29466.

15. Leite NC, Salles GF, Araujo AL, Villela-Nogueira CA, Cardoso CR. Prevalence and associated factors of non-alco- 
holic fatty liver disease in patients with type-2 diabetes mellitus. Liver Int 2009;29:113-119.

16. Kim D, Kim WR, Kim HJ, Therneau TM. Association between noninvasive fibrosis markers and mortality among adults with nonalcoholic fatty liver disease in the United States. Hepatology 2013;57:1357-1365.

17. Hsu CS, Kao JH. Non-alcoholic fatty liver disease: an emerging liver disease in Taiwan. J Formos Med Assoc 2012;111:527-535.

18. Chen J, Talwalkar JA, Yin M, Glaser KJ, Sanderson SO, Ehman RL. Early detection of nonalcoholic steatohepatitis in patients with nonalcoholic fatty liver disease by using MR elastography. Radiology 2011;259:749-756.

19. Kim D, Kim WR, Talwalkar JA, Kim HJ, Ehman RL. Advanced fibrosis in nonalcoholic fatty liver disease: noninvasive assessment with MR elastography. Radiology 2013;268:411-419.

20. Nobili V, Vizzutti F, Arena U, et al. Accuracy and reproducibility of transient elastography for the diagnosis of fibrosis in pediatric nonalcoholic steatohepatitis. Hepatology 2008;48:442-448.

21. Wong VW, Vergniol J, Wong GL, et al. Diagnosis of fibrosis and cirrhosis using liver stiffness measurement in nonalcoholic fatty liver disease. Hepatology 2010;51:454462.

22. McPherson S, Stewart SF, Henderson E, Burt AD, Day CP. Simple non-invasive fibrosis scoring systems can reliably exclude advanced fibrosis in patients with non-alcoholic fatty liver disease. Gut 2010;59:1265-1269.

23. Musso G, Gambino R, Cassader M, Pagano G. Meta-analysis: natural history of non-alcoholic fatty liver disease (NAFLD) and diagnostic accuracy of non-invasive tests for liver disease severity. Ann Med 2011;43:617-649.

24. Shah AG, Lydecker A, Murray K, et al. Comparison of noninvasive markers of fibrosis in patients with nonalcoholic fatty liver disease. Clin Gastroenterol Hepatol 2009;7:1104-1112.

25. Hamaguchi M, Kojima T, Takeda N, et al. Nonalcoholic fatty liver disease is a novel predictor of cardiovascular disease. World J Gastroenterol 2007;13:1579-1584.

26. Kim D, Choi SY, Park EH, et al. Nonalcoholic fatty liver disease is associated with coronary artery calcification. Hepatology 2012;56:605-613.

27. Park HE, Kwak MS, Kim D, Kim MK, Cha MJ, Choi SY. Nonalcoholic fatty liver disease is associated with coronary artery calcification development: a longitudinal study. J Clin Endocrinol Metab 2016;101:3134-3143.

28. Valenti L, Bugianesi E, Pajvani U, Targher G. Nonalcoholic fatty liver disease: cause or consequence of type 2 diabetes? Liver Int 2016;36:1563-1579.

29. Goessling W, Massaro JM, Vasan RS, D'Agostino RB Sr, Ellison RC, Fox CS. Aminotransferase levels and 20-year risk of metabolic syndrome, diabetes, and cardiovascular disease. Gastroenterology 2008;135:1935-1944, 1944.

30. Lonardo A, Ballestri S, Marchesini G, Angulo P, Loria P. Nonalcoholic fatty liver disease: a precursor of the metabolic syndrome. Dig Liver Dis 2015;47:181-190.

31. Calori G, Lattuada G, Ragogna F, et al. Fatty liver index and mortality: the Cremona study in the 15th year of follow-up. Hepatology 2011;54:145-152.

32. Guturu P, Duchini A. Etiopathogenesis of nonalcoholic steatohepatitis: role of obesity, insulin resistance and mechanisms of hepatotoxicity. Int J Hepatol 2012;2012:212865.

33. Kim D, Kim WR. Nonobese fatty liver disease. Clin Gastroenterol Hepatol 2017;15:474-485.

34. Armstrong MJ, Adams LA, Canbay A, Syn WK. Extrahepatic complications of nonalcoholic fatty liver disease. Hepatology 2014;59:1174-1197.

35. Targher G, Day CP, Bonora E. Risk of cardiovascular disease in patients with nonalcoholic fatty liver disease. $\mathrm{N}$ Engl J Med 2010;363:1341-1350.

36. European Association for the Study of the Liver (EASL); European Association for the Study of Diabetes (EASD); European Association for the Study of Obesity (EASO). EASL-EASD-EASO clinical practice guidelines for the management of non-alcoholic fatty liver disease. J Hepatol 2016;64:1388-1402.

37. Panagiotakos DB, Pitsavos C, Chrysohoou C, et al. Impact of lifestyle habits on the prevalence of the metabolic syndrome among Greek adults from the ATTICA study. Am Heart J 2004;147:106-112.

38. Dalle Grave R, Calugi S, Centis E, Marzocchi R, El Ghoch M, Marchesini G. Lifestyle modification in the management of the metabolic syndrome: achievements and challenges. Diabetes Metab Syndr Obes 2010;3:373-385.

39. Perez-Martinez P, Mikhailidis DP, Athyros VG, et al. Lifestyle recommendations for the prevention and management of metabolic syndrome: an international panel recommendation. Nutr Rev 2017;75:307-326.

40. Vilar-Gomez E, Martinez-Perez Y, Calzadilla-Bertot L, et al. Weight loss through lifestyle modification significantly reduces features of nonalcoholic steatohepatitis. Gas- 
troenterology 2015;149:367-378.e5.

41. Orci LA, Gariani K, Oldani G, Delaune V, Morel P, Toso C. Exercise-based interventions for nonalcoholic fatty liver disease: a meta-analysis and meta-regression. Clin Gastroenterol Hepatol 2016;14:1398-1411.

42. Katsagoni CN, Georgoulis M, Papatheodoridis GV, Panagiotakos DB, Kontogianni MD. Effects of lifestyle interventions on clinical characteristics of patients with non-alcoholic fatty liver disease: a meta-analysis. Metabolism 2017;68:119-132.

43. Oh S, Shida T, Yamagishi K, et al. Moderate to vigorous physical activity volume is an important factor for managing nonalcoholic fatty liver disease: a retrospective study. Hepatology 2015;61:1205-1215.

44. Thoma C, Day CP, Trenell MI. Lifestyle interventions for the treatment of non-alcoholic fatty liver disease in adults: a systematic review. J Hepatol 2012;56:255-266.

45. Keating SE, Hackett DA, George J, Johnson NA. Exercise and non-alcoholic fatty liver disease: a systematic review and meta-analysis. J Hepatol 2012;57:157-166.

46. Thompson PD, Buchner D, Pina IL, et al. Exercise and physical activity in the prevention and treatment of atherosclerotic cardiovascular disease: a statement from the Council on Clinical Cardiology (Subcommittee on Exercise, Rehabilitation, and Prevention) and the Council on Nutrition, Physical Activity, and Metabolism (Subcommittee on Physical Activity). Circulation 2003;107:31093116.

47. Pate RR, Pratt M, Blair SN, et al. Physical activity and public health. A recommendation from the Centers for Disease Control and Prevention and the American College of Sports Medicine. JAMA 1995;273:402-407.

48. Fletcher GF, Balady GJ, Amsterdam EA, et al. Exercise standards for testing and training: a statement for healthcare professionals from the American Heart Association. Circulation 2001;104:1694-1740.

49. Craig CL, Marshall AL, Sjostrom M, et al. International physical activity questionnaire: 12-country reliability and validity. Med Sci Sports Exerc 2003;35:1381-1395.

50. Hashida R, Kawaguchi T, Bekki M, et al. Aerobic vs. resistance exercise in non-alcoholic fatty liver disease: a systematic review. J Hepatol 2017;66:142-152.

51. Kistler KD, Brunt EM, Clark JM, et al. Physical activity recommendations, exercise intensity, and histological severity of nonalcoholic fatty liver disease. Am J Gastroenterol 2011;10:460-468.
52. Houghton D, Thoma C, Hallsworth K, et al. Exercise reduces liver lipids and visceral adiposity in patients with nonalcoholic steatohepatitis in a randomized controlled trial. Clin Gastroenterol Hepatol 2017;15:96-102.

53. Bacchi E, Negri C, Targher G, et al. Both resistance training and aerobic training reduce hepatic fat content in type 2 diabetic subjects with nonalcoholic fatty liver disease (the RAED2 Randomized Trial). Hepatology 2013;58:1287-1295.

54. Shamsoddini A, Sobhani V, Ghamar Chehreh ME, Alavian SM, Zaree A. Effect of aerobic and resistance exercise training on liver enzymes and hepatic fat in iranian men with nonalcoholic fatty liver disease. Hepat Mon 2015;15:e31434.

55. Slentz CA, Bateman LA, Willis LH, et al. Effects of aerobic vs. resistance training on visceral and liver fat stores, liver enzymes, and insulin resistance by HOMA in overweight adults from STRRIDE AT/RT. Am J Physiol Endocrinol Metab 2011;301:E1033-E1039.

56. Hallsworth K, Fattakhova G, Hollingsworth KG, et al. Resistance exercise reduces liver fat and its mediators in non-alcoholic fatty liver disease independent of weight loss. Gut 2011;60:1278-1283.

57. Koo BK, Kim D, Joo SK, et al. Sarcopenia is an independent risk factor for non-alcoholic steatohepatitis and significant fibrosis. J Hepatol 2017;66:123-131.

58. Keating SE, Hackett DA, Parker HM, et al. Effect of aerobic exercise training dose on liver fat and visceral adiposity. J Hepatol 2015;63:174-182.

59. Ryu S, Chang Y, Jung HS, et al. Relationship of sitting time and physical activity with non-alcoholic fatty liver disease. J Hepatol 2015;63:1229-1237.

6o. Kwak MS, Kim D, Chung GE, Kim W, Kim YJ, Yoon JH. Role of physical activity in nonalcoholic fatty liver disease in terms of visceral obesity and insulin resistance. Liver Int 2015;35:944-952.

61. Hickman IJ, Byrne NM, Croci I, et al. A pilot randomised study of the metabolic and histological effects of exercise in non-alcoholic steatohepatitis. J Diabetes Metab 2013;4:300.

62. Sreenivasa Baba C, Alexander G, Kalyani B, et al. Effect of exercise and dietary modification on serum aminotransferase levels in patients with nonalcoholic steatohepatitis. J Gastroenterol Hepatol 2006;21(1 Pt 1):191-198.

63. Keating SE, George J, Johnson NA. The benefits of exercise for patients with non-alcoholic fatty liver disease. 
Expert Rev Gastroenterol Hepatol 2015;9:1247-1250.

64. Barengo NC, Antikainen R, Borodulin K, Harald K, Jousi-

lahti P. Leisure-time physical activity reduces total and cardiovascular mortality and cardiovascular disease incidence in older adults. J Am Geriatr Soc 2017;65:504-510.

65. Barengo NC, Hu G, Lakka TA, Pekkarinen H, Nissinen A, Tuomilehto J. Low physical activity as a predictor for total and cardiovascular disease mortality in middle-aged men and women in Finland. Eur Heart J 2004;25:2204-2211.

66. Mora S, Redberg RF, Cui Y, et al. Ability of exercise testing to predict cardiovascular and all-cause death in asymptomatic women: a 20-year follow-up of the lipid research clinics prevalence study. JAMA 2003;290:1600-1607.

67. Ekelund LG, Haskell WL, Johnson JL, Whaley FS, Criqui $\mathrm{MH}$, Sheps DS. Physical fitness as a predictor of cardiovascular mortality in asymptomatic North American men. The Lipid Research Clinics Mortality Follow-up Study. N Engl J Med 1988;319:1379-1384.

68. Kodama S, Saito K, Tanaka S, et al. Cardiorespiratory fitness as a quantitative predictor of all-cause mortality and cardiovascular events in healthy men and women: a meta-analysis. JAMA 2009;301:2024-2035.

69. Knowler WC, Barrett-Connor E, Fowler SE, et al. Reduction in the incidence of type 2 diabetes with lifestyle intervention or metformin. N Engl J Med 2002;346:393-403.

70. Fagard RH. Exercise characteristics and the blood pres- sure response to dynamic physical training. Med Sci Sports Exerc 2001;33(6 Suppl):S484-S492.

71. Thompson PD, Crouse SF, Goodpaster B, Kelley D, Moyna N, Pescatello L. The acute versus the chronic response to exercise. Med Sci Sports Exerc 2001;33(6 Suppl):S438-S445.

72. Couillard C, Despres JP, Lamarche B, et al. Effects of endurance exercise training on plasma HDL cholesterol levels depend on levels of triglycerides: evidence from men of the Health, Risk Factors, Exercise Training and Genetics (HERITAGE) Family Study. Arterioscler Thromb Vasc Biol 2001;21:1226-1232.

73. Kwak MS, Kim D, Chung GE, Kim W, Kim JS. The preventive effect of sustained physical activity on incident nonalcoholic fatty liver disease. Liver Int 2017;37:919-926.

74. Sung KC, Ryu S, Lee JY, Kim JY, Wild SH, Byrne CD. Effect of exercise on the development of new fatty liver and the resolution of existing fatty liver. J Hepatol 2016;65:791-797.

75. Tsunoda K, Kai Y, Kitano N, Uchida K, Kuchiki T, Nagamatsu T. Impact of physical activity on nonalcoholic steatohepatitis in people with nonalcoholic simple fatty liver: a prospective cohort study. Prev Med 2016;88:237240.

76. Jan CF, Nfor ON, Huang JY, et al. Exercise might prevent cirrhosis in overweight and obese adults. Liver Int 2017 Aug 19 [Epub]. https://doi.org/10.1111/liv.13553. 